\title{
Prevenção primária em saúde na adolescência: avaliação de um
Prom programa de habilidades de vida
}

\author{
Sheila Giardini Murta \\ Universidade de Brasília \\ Francimara Azevedo Borges \\ Danilo Cruvinel Ribeiro \\ Eliana Porto Rocha \\ Jordana Calil Lopes de Menezes \\ Pontificia Universidade Católica de Goiás \\ Marina de Moraes e Prado \\ Pontifícia Universidade Católica do Rio Grande do Sul
}

\begin{abstract}
Resumo
Este artigo descreve o processo e resultados alcançados em um programa de habilidades de vida para dezoito adolescentes, de uma amostra não clínica, entre 12 a 14 anos de idade. Dezesseis sessões de grupo foram feitas, baseadas em uma abordagem psico-educativa e em técnicas de grupo. A avaliação de processo incluiu medidas diárias de satisfação do consumidor, prática de habilidades treinadas no curso da intervenção e suporte fornecido pelo grupo. A avaliação de processo demonstrou que os participantes se sentiram satisfeitos com o programa; praticaram habilidades sociais, habilidades cognitivas e habilidades de manejo de estresse aprendidas durante a intervenção; e demonstram um senso de confiança na intervenção em grupo. Entrevistas de follow-up conduzidas um ano depois com os adolescentes e seus pais mostraram que as mudanças comportamentais se mantiveram. Os resultados mostraram-se positivos, mas estudos experimentais serão necessários para conclusões definitivas sobre a efetividade do programa.
\end{abstract}

Palavras-chave: habilidades de vida; habilidades sociais; prevenção primária; estudo de intervenção; avaliação de processo.

\begin{abstract}
Primary prevention in health in adolescence: evaluation of a life skills program. This paper describes the process and results reached in a life skills program for 18 adolescents, from a non-clinical sample, with 12 to 14 years-old. Sixteen group sessions were done, based on a psycho-educational approach and group techniques. The process evaluation included diary measures of consumer satisfaction, practice of skills trained in the course of the intervention and support provided by the group. The process evaluation demonstrated that the participants felt satisfied with the program; practiced social skills, cognitive skills and stress management skills learned during the intervention; and demonstrated a sense of confidence in the group intervention. Follow-up interviews one year after the intervention conducted with the adolescents and their parents shown that the behavioral changes were maintained. The results suggest positive results from the intervention, but experimental studies will be necessary for robust conclusions on the effectiveness of this program.
\end{abstract}

Keywords: life skills; social skills; primary prevention; intervention study; process evaluation.

A promoção de saúde entre os adolescentes tem se tornado uma necessidade imperativa. A alta incidência de gravidez precoce e indesejada, o abuso de álcool e outras drogas (Hawkins, Catalano, \& Miller, 1992; Murta, Del Prette, Nunes, \& Del Prette, 2007), o envolvimento em acidentes de trânsito, a delinqüência, as doenças sexualmente transmissíveis
(Straub, 2005), os transtornos de humor (Essau, 2002) e o suicídio (McCarter, Sowers, \& Dulmus, 2004; Schochet et al., 2001) são alguns dos indicadores que sugerem ser este um grupo que precisa de cuidados preventivos para com sua saúde. De modo geral, contribuem para estes problemas o engajamento em comportamentos de risco, tais como manter relações sexuais sem 
anticonceptivos, usar seringas contaminadas, aderir a grupos de amigos com comportamentos anti-sociais, comportar-se de modo violento e impulsivo e dirigir alcoolizado. O desenvolvimento e a avaliação de programas preventivos para tais comportamentos são de grande relevância, por evitar ou reduzir as perdas e custos decorrentes destes problemas para o adolescente, para sua família, para o sistema de saúde, para a sociedade e para as gerações futuras.

Dentre as diversas abordagens de prevenção a comportamentos de risco à saúde entre adolescentes, encontramse os programas de ensino de habilidades de vida. O termo habilidades de vida designa um conjunto de habilidades necessárias ao enfrentamento saudável às demandas cotidianas, dentre as quais se incluem habilidades para a tomada de decisão, resolução de problemas, pensamento crítico, pensamento criativo, comunicação eficaz, relacionamento interpessoal, autoconhecimento, empatia, manejo de emoções e de estresse (Gorayeb, 2002). Diversos estudos têm evidenciado a efetividade de programas desta natureza na redução dos comportamentos de risco e no fortalecimento de competências associadas à saúde entre adolescentes e jovens (Botvin, 1996; Gorayeb, Cunha Netto, \& Bugliani, 2003; Mangrulkar, Whitman, \& Posner, 2001; Minto, Pedro, Cunha Netto, Bugliani, \& Gorayeb, 2006). Tendo em vista a efetividade de programas de ensino de habilidades de vida, a Organização Mundial de Saúde tem recomendado este tipo de programa como uma das estratégias preferenciais de prevenção e promoção de saúde nesta etapa de vida.

Programas focados em habilidades de vida são teoricamente embasados na teoria da aprendizagem social e em estudos sobre resiliência, fatores de risco e fatores de proteção no curso do desenvolvimento (Mangrulkar et al., 2001; Meschke \& Patterson, 2003). Adotam o pressuposto de que o uso de metodologias grupais, vivenciais e interativas possibilita o desenvolvimento de fatores de proteção associados a competências individuais para lidar com situações de risco, incluindo habilidades de enfrentamento ao estresse (como habilidades para lidar com crises), habilidades cognitivas (como pensamento conseqüencial) e habilidades sociais (como assertividade e empatia) (Spence, 2003). Estas habilidades permitem ao adolescente enfrentar de modo mais saudável as situações de risco à sua saúde, advindas de possíveis conflitos ou pressões vivenciados nas relações com familiares, professores, parceiros e pares. Os programas de habilidades de vida são multicomponentes, isto é, agregam conteúdos e técnicas diversificados e são de longa ou média duração. Em função de tais características metodológicas, quando comparados às abordagens exclusivamente informativas comumente empregadas no cotidiano das escolas e da comunidade, estes programas teriam mais chances de produzir e manter mudanças comportamentais entre adolescentes. Dentre estas mudanças, destacam-se o desenvolvimento de autoconhecimento e identidade, de comunicação assertiva de sentimentos e opiniões, de tomada de decisão coerente com projeto de vida, de busca da rede de apoio social e do não engajamento em comportamentos aditivos como forma de alívio de emoções desagradáveis.

No Brasil, estudos pioneiros sobre o ensino de habilidades de vida vêm sendo feitos pelo Programa de Promoção de Saúde na Comunidade do Hospital das Clínicas da Universidade de São Paulo, em Ribeirão Preto (Gorayeb, 2002). Em uma de suas primeiras versões, o programa foi direcionado a grupos de adolescentes e professores em escolas, com 12 a 20 sessões grupais, de 90 minutos de duração, em encontros semanais. Foram empregadas dinâmicas de grupo, exercícios de aquecimento, dramatizações, exposição oral e discussões. Avaliações feitas através do relato verbal dos participantes evidenciaram melhora na interação grupal, melhora na interação dos participantes com o facilitador do grupo, melhora no estabelecimento de relações interpessoais fora do grupo e aumento de consciência sobre situações de risco e habilidades requeridas para seu manejo adequado (Gorayeb et al., 2003). Os resultados deste estudo estão em acordo com os resultados de revisões da literatura especializada em prevenção primária em saúde na infância e adolescência (Durlak \& Wells, 1997; Kulic, Horne \& Dagley, 2004; Murta, 2007a), que apontam a efetividade de programas preventivos para crianças e adolescentes.

Além das evidências de efetividade, estas revisões apontam uma agenda para o avanço das pesquisas em programas preventivos, como o estabelecimento de metas e procedimentos claros, a avaliação longitudinal de efeitos do programa, a verificação de como os resultados do programa se relacionam a características individuais dos participantes e da intervenção, e a avaliação do processo de implementação dos programas. De fato, a avaliação de processo de programas em saúde tem sido considerada indispensável para a identificação dos fatores responsáveis pelo sucesso ou fracasso do programa, fornecendo informações cruciais para a interpretação dos seus resultados finais (Posavac \& Carey, 2003; Steckler \& Linnan, 2002). Este tipo de avaliação, também conhecido como avaliação formativa ou avaliação de monitoramento (Fernández-Ballesteros, Vedung, \& Seyfried, 1998) pode elucidar em que extensão variáveis outras que não o conteúdo da intervenção, tais como o sucesso do recrutamento, fatores contextuais facilitadores ou dificultadores da implantação da intervenção, a assimilação e uso das ferramentas disponibilizadas pela intervenção, a satisfação dos participantes para com o programa, o senso de segurança e confiança na intervenção podem afetar as mudanças nas metas finais do programa, imediatamente ao final do programa e ao longo do tempo (Murta, Sanderson, \& Oldenburg, 2007).

Seguindo estas recomendações acerca da metodologia de avaliação de programas preventivos na infância e adolescência, o presente estudo consiste na descrição da avaliação de processo e de resultados alcançados em um programa de ensino de habilidades de vida conduzido em um grupo de adolescentes. Este estudo teve como propósito responder a dois grupos de questões: o primeiro relativo à avaliação de processo do programa, e o segundo relativo aos resultados do programa. Com relação à avaliação de processo do programa, os objetivos específicos foram: (a) avaliar o alcance de metas intermediárias previstas para o programa, sendo estas os seguintes comportamentos manifestos durante as sessões: relatar problemas, relatar progressos, chorar, explicar causas do próprio comportamento e fornecer apoio ao colega; (b) descrever a dose recebida, definida como a prática de ferramentas disponibilizadas pelo programa e (c) avaliar a satisfação do consumidor, neste estudo entendida 
como auto-relatos de sentimentos sobre a sessão ocorridos em cada encontro. Com relação à avaliação de resultados do programa, os objetivos específicos foram: (a) identificar a manutenção ou perda das habilidades aprendidas no programa um ano após o seu término, de acordo com a percepção dos adolescentes e seus pais e (b) descrever a ocorrência de eventos de saúde relevantes, incluindo o uso de drogas, gravidez precoce, desempenho escolar e doenças sexualmente transmissíveis, conforme a percepção dos adolescentes e seus pais, no período de um ano depois da intervenção.

\section{Método}

\section{Delineamento e Participantes}

Foi utilizado um delineamento pré-experimental (Campbell \& Stanley, 1979) e avaliação de follow-up, feita um ano após o término do programa. Participaram do estudo dezoito adolescentes provenientes de famílias de funcionários docentes e administrativos de uma universidade do centro-oeste brasileiro. Todos residiam com os pais, cujo nível de instrução variava entre ensino médio e ensino superior. Os adolescentes, na época de inscrição no programa, tinham entre 12 e 14 anos e cursavam entre o $7^{\circ}$ ano do ensino fundamental e o primeiro do ensino médio. Um deles havia repetido o ano escolar e aproximadamente metade deles estudava em escola pública. Três deles relataram ter algum problema de saúde física, que não requeria o uso de medicamentos controlados no tratamento. Todos os adolescentes relataram não fazer uso de fumo, álcool e drogas ilegais. Aproximadamente metade deles praticava algum esporte e participava de algum grupo, e dois relataram ter namorado(a). Eram, em sua maioria, do sexo feminino (15 meninas e 3 meninos).

Os critérios para inclusão no programa foram: ter entre 12 e 14 anos, ser estudante e desejar participar voluntariamente do programa. Esta faixa etária foi escolhida em função de ser o início da adolescência e, portanto, o momento ideal para a prevenção de problemas cuja ocorrência em geral se dá na adolescência média ou tardia. Os critérios para exclusão foram apresentar experiência pessoal com uso de drogas, transtornos mentais e gravidez.

\section{Ambiente, material e instrumentos}

O programa foi realizado no espaço da universidade, em uma sala usada para desenvolvimento de pessoal, vinculada ao departamento de recursos humanos da instituição. Os materiais usados na realização da intervenção foram: material de consumo (como lápis de cor, folhas de papel, argila, tinta, balões, dentre outros) e material de uso permanente (como CDs para relaxamento, televisão e vídeo). Os instrumentos para avaliação do programa foram:

Questionário Sociodemográfico. Composto por 21 questões fechadas sobre características demográficas e de saúde do adolescente e da família. As questões abrangiam a idade, sexo, escolarização, saúde, participação em atividades grupais e esportivas, instrução e ocupação dos pais.

Checklist de Metas Intermediárias e Atitudes frente à Intervenção (Murta, 2007b). Apresenta uma lista de comportamentos indicadores do "clima da sessão", cuja ocorrência pode ser interpretada como favorável para a efetividade do programa e sugestiva de um clima de confiança e apoio mútuo entre os participantes e entre estes e os facilitadores do grupo. O instrumento é composto pelas seguintes categorias comportamentais: relatar problemas, relatar progressos, chorar, explicar causas do próprio comportamento e oferecer ajuda a colegas. A ocorrência destes comportamentos era registrada em cada sessão, independente de quem emitiu o comportamento e quantas vezes o fez na sessão.

Folha para Registro de Dose Recebida. Tinha por objetivo registrar os relatos dos participantes sobre as habilidades ensinadas no programa que haviam sido praticadas por eles na semana anterior. Tratava-se de uma ficha de uso diário, para cada sessão.

Folha para Registro de Satisfação. Foi utilizada para registrar os relatos dos sentimentos vivenciados em cada sessão, indicadores de satisfação ou insatisfação frente à sessão do dia.

Roteiro de Entrevista para Adolescentes e Pais. Consistia em um roteiro de entrevista semi-estruturada, conduzida durante visita domiciliar um ano após o término do programa, com 23 questões acerca de como os adolescentes e seus pais percebiam a manutenção das habilidades aprendidas no programa e o impacto do programa no cotidiano do adolescente. A parte dirigida ao adolescente continha dezesseis questões sobre lembranças acerca do programa, ferramentas ensinadas pelo programa ainda praticadas pelo adolescente, mudanças percebidas como decorrentes da participação no programa, desempenho escolar, saúde e sugestões para melhoria do programa. A parte dirigida aos pais continha sete questões sobre a percepção dos pais sobre as mudanças comportamentais dos filhos após a participação no programa, o estado de saúde e o desempenho escolar do adolescente e sugestões para melhoria do programa.

\section{Procedimentos}

Recrutamento e cuidados éticos. Os participantes em potencial foram convidados a participar através de uma carta dirigida ao endereço residencial dos funcionários da universidade. A carta era dirigida à família e apresentava, em linguagem não técnica, os objetivos, formato, conteúdo do programa, a equipe responsável pela intervenção-pesquisa e era assinada pelo diretor de recursos humanos da universidade. Os interessados se inscreveram por telefone no departamento de recursos humanos da instituição e foram convidados, adolescentes e responsáveis, a participar de uma reunião para esclarecimento de dúvidas e de aspectos éticos. Nesta reunião, os que desejaram participar do programa expressaram seu desejo e concordância assinando o Termo de Consentimento Livre e Esclarecido. Este estudo foi aprovado pelo Comitê de Ética da Universidade Católica de Goiás.

Programa de intervenção. A intervenção foi em grupo, do tipo psico-educativo, conduzida em dezesseis sessões semanais, ao longo de cinco meses, com duração de 90 minutos cada sessão, num total de 24 horas de intervenção. A intervenção foi implementada por uma dupla de facilitadores, composta por 
alunos de graduação em Psicologia, previamente treinados. O treinamento foi feito por meio de cinco estratégias de ensino: (a) estudo teórico da literatura especializada; (b) exposição direta ao procedimento de intervenção, quando os alunos foram "usuários" de um grupo de testagem das técnicas e procedimentos previstos para a intervenção com os adolescentes. Este grupo foi conduzido pela primeira autora, com experiência anterior na condução de grupos psico-educativos, e funcionou como um grupo real de intervenção; (c) a atuação da facilitadora no grupo de testagem foi filmada para posterior estudo por parte dos alunos que atuariam como facilitadores nos grupos; (d) estudo de um manual contendo os objetivos, materiais e procedimentos de cada sessão da intervenção (Murta, 2008) e (e) supervisão para discussão das sessões de intervenção já conduzidas. Os facilitadores foram encorajados a desenvolver habilidades comunicativas capazes de gerar entre os participantes a identificação de ganhos e perdas decorrentes da própria ação, recursos pessoais para enfrentamento, auto-aceitação e um senso de segurança e confiança no grupo.

Os adolescentes participantes foram alocados em três grupos, de aproximadamente 6 participantes cada. O procedimento utilizado na íntegra está descrito em Murta (2008). Em síntese, foram discutidos os temas autoconceito, auto-eficácia, diferenciação entre riscos para o crescimento pessoal e riscos vazios; relação crenças, emoções e comportamento; comunicação assertiva; empatia; resolução de problemas; enfrentamento ao estresse; tomada de decisão; pensamento crítico e riscos à saúde associados ao gênero; locus de controle interno e saúde; rede de apoio social e prevenção de recaída. Estes temas foram trabalhados por meio de vivências grupais (Serrão \& Baleeiro, 1999), técnicas de treinamento em habilidades sociais (Del Prette \& Del Prette, 2001), criatividade (Virgolim, Fleith, \& Neves-Pereira, 1999), arteterapia (Liebman, 2000) e técnicas cognitivo-comportamentais, incluindo ensaio comportamental, relaxamento, reestruturação cognitiva, solução de problemas, modelagem e tarefas de casa (Stallard, 2004). Cada sessão seguiu a seguinte rotina: verificação da prática de habilidades ou avaliação de "dose recebida", introdução ao tema do dia, vivência ou técnica principal, discussão da vivência ou técnica principal, oferecimento de informação, tarefa de casa e avaliação de satisfação frente à sessão ou avaliação de "satisfação do consumidor".

O programa constou ainda de dois encontros com os pais dos adolescentes, com quatro horas de duração cada, quando foram discutidas práticas educativas parentais promotoras de autonomia na adolescência (Gomide, 2003), incluindo o uso de habilidades sociais educativas nas interações pais e filhos (Bolsoni-Silva \& Marturano, 2002; Coelho \& Murta, 2007; Pinheiro, Haase, Del Prette, Amarente, \& Del Prette, 2006). Estas sessões foram conduzidas por uma psicóloga com experiência em treinamento de pais e consistiram na realização de vivências grupais, de modo similar àquelas conduzidas com os adolescentes. Além disto, foi incentivada a troca de experiências e suporte social entre os pais.

\section{Avaliação e análise de dados}

Avaliação de processo. A partir da terceira sessão, imediatamente ao final de cada sessão, os facilitadores preenchiam o Checklist de Metas Intermediárias e Atitudes frente à Intervenção, a Folha para Registro de Dose Recebida e a Folha para Registro de Satisfação do Consumidor. Este preenchimento era antecedido pela discussão entre a dupla de facilitadores dos principais eventos ocorridos na sessão e, em seguida, eram registrados aqueles sobre os quais a dupla estava em acordo. $\mathrm{O}$ registro imediatamente após a sessão foi feito para se preservar ao máximo a integridade do dado, e ao mesmo tempo disponibilizar os facilitadores para interagir com o grupo durante a intervenção, sem a tarefa concorrente de efetuar registros durante a sessão. Os dados relativos ao Checklist de Metas Intermediárias e Atitudes frente à Intervenção foram quantificados, de modo a se formar índices de freqüência de ocorrência. Os dados verbais coletados por meio das Folhas de Registro de Dose Recebida e de Satisfação do Consumidor foram analisados através de análise de conteúdo.

Avaliação de follow-up. Um ano após o término do programa, os adolescentes e seus pais foram entrevistados em seus domicílios, quando foi utilizado o Roteiro de Entrevista para Adolescentes e Pais. A entrevista foi feita conjuntamente, com a presença dos pais e filhos. Os adolescentes e seus pais foram indagados acerca dos principais eventos ocorridos na vida do adolescente após o programa, a saúde do adolescente neste período, a percepção acerca do desempenho escolar, o uso das habilidades aprendidas na intervenção e as mudanças comportamentais percebidas como facilitadas pelo programa. As verbalizações foram gravadas em áudio e posteriormente submetidas à categorização.

\section{Resultados e Discussão}

\section{Avaliação de processo}

A observação direta e sistemática da ocorrência das metas intermediárias durante as sessões de intervenção mostrou que todas as metas ocorreram, por ordem de freqüência de ocorrência: relatar problemas pessoais, relatar progressos pessoais, explicar causas do próprio comportamento, fornecer apoio ao colega e chorar. As freqüências de ocorrência de cada meta intermediária estão disponíveis na Tabela 1. Estes dados evidenciam que os comportamentos cuja ocorrência ao longo da intervenção foi considerada favorável à efetividade do programa se manifestaram, numa freqüência satisfatória, ao longo do programa. A ocorrência destas metas intermediárias indica que os participantes fizeram auto-revelação de problemas e mudanças positivas em seus desempenhos, identificaram as causas de seus comportamentos, se apoiaram e manifestaram sentimentos. Considerando que o relato de problemas pessoais foi a meta intermediária que mais ocorreu (41 ocorrências), e que o oferecimento de apoio a colegas ocorreu em um pouco mais da metade das ocasiões possíveis ( 24 ocorrências em 42 possíveis), pode-se supor que esta última poderia ter sido mais encorajada entre os participantes, em especial nas ocasiões em que havia relatos de problemas pessoais. Isto poderia ter fortalecido ainda mais o clima de suporte e confiança no grupo. Apesar disto, considera-se que estas evidências sugerem um clima do grupo 
suficientemente seguro, apoiador e amistoso, uma vez que houve uma alta ocorrência de relatos de problemas pessoais (41), bem como progressos pessoais (40) e algumas ocorrências de choro (7). Além disto, a ocorrência de explicações do próprio comportamento (33) sugere que o grupo pode ter funcionado como fonte de reatribuição de significados dos eventos vividos, o que pode também implicar em mudanças em estratégias de enfrentamento, seja pela via cognitiva (como deixar de perceberse inadequado ou extravagante após entender as causas do próprio comportamento) seja pela via comportamental (como mudar a própria conduta, através da mudança das causas passíveis de controle pelo participante) (Straub, 2005).

Tabela 1

Freqüência de ocorrência de metas intermediárias*

\begin{tabular}{lc}
\hline Metas intermediárias & Freqüência \\
\hline Relatar problemas pessoais & 41 \\
Relatar progressos pessoais & 40 \\
Chorar na sessão & 7 \\
Explicar causas do próprio comportamento & 33 \\
Fornecer apoio ao colega & 24 \\
\hline Total & 145 \\
* Nota: total de ocorrências possíveis: & 42 \\
observadas em 3 grupos de intervenção).
\end{tabular}

A análise dos relatos verbais dos participantes acerca do uso das ferramentas disponibilizadas pelo programa mostrou uma grande diversidade de habilidades praticadas, categorizadas em habilidades sociais ( 91 relatos de prática), habilidades cognitivas (62) e enfrentamento ao estresse (47). Na categoria prática de habilidades sociais, as habilidades mais freqüentemente praticadas foram: assertividade/dizer não, negociar/argumentar com os pais, solicitar/dar apoio, demonstrar empatia, fazer amizades, reconciliar/pedir desculpas, fazer amizades, iniciar conversa e controlar raiva e impulsividade. Na categoria prática de habilidades cognitivas, as habilidades mais praticadas foram planejamento de passos para solução de problemas, planejamento do futuro como homem/mulher, fortalecimento de auto-eficácia e mudança em erros de pensamento. Na categoria enfrentamento ao estresse, foram mais freqüentes relatos de uso de estratégias de enfrentamento focadas no problema (como adotar comportamentos de cuidados com a saúde) do que focadas na emoção (como respirar profundamente). As categorias e subcategorias de habilidades praticadas e suas respectivas freqüências e porcentagens estão apresentadas na Tabela 2.

Tomando-se em consideração estes dados, foi constatada uma boa "dose recebida", isto é, os recursos disponibilizados pelo programa foram assimilados e utilizados pelos adolescentes em seu cotidiano. Tal prática de habilidades oferecidas pelo programa no decorrer da intervenção é considerada por Steckler e Linnan (2002) um dos principais precursores do alcance das metas finais planejadas para programas em saúde.

A análise dos relatos dos participantes sobre os sentimentos experimentados na sessão do dia, indicadores de satisfação ou insatisfação com a sessão, resultou em cinco categorias de sentimentos acerca da sessão: alegre/feliz/contente (exemplo: por estar aprendendo e fazendo descobertas), pensativo (ex.: pelos assuntos discutidos), preocupado (ex.: com as decisões a serem tomadas), relaxado/aliviado (ex.: por expressar os sentimentos) e triste (ex.: por falar do mau relacionamento dos pais).

A diversidade dos sentimentos experimentados, incluindo o estar contente e estar relaxado, sugere estados de satisfação para com a intervenção, o que se torna mais evidente pela análise das razões para se sentir contente e relaxado, como estar se soltando no grupo e poder desabafar e falar sobre tudo. A ocorrência de sentimentos desagradáveis, como tristeza e preocupação, não evidencia necessariamente insatisfação para com a intervenção, a não ser pelo tempo considerado curto para poder se colocar em profundidade e participar da sessão. Mesmo os sentimentos desagradáveis, como estar triste por se descobrir não assertivo ou preocupado com os enfrentamentos necessários, podem ser úteis e produtivos em um processo de mudança. De acordo com Kholenberg e Tsai (2001), entrar em contato com as emoções é um passo necessário para entrar em contato com as contingências e então identificar o que pode ser mudado e efetuar as mudanças possíveis.

Em síntese, os relatos de sentimentos vivenciados em cada sessão apontam uma predominância de experiências de satisfação do que insatisfação frente à intervenção. Contudo, há que se considerar que os poucos relatos sobre insatisfação com a intervenção podem também estar associados ao procedimento de avaliação de satisfação escolhido neste estudo. Ao invés de se usar perguntas mais gerais sobre sentimentos vividos na sessão, perguntas mais diretas do tipo "o que vocês gostaram ou não gostaram na sessão de hoje”, poderiam gerar dados mais específicos sobre insatisfação.

\section{Avaliação de follow-up}

Treze dos dezoito adolescentes e seus pais foram localizados e aceitaram participar da avaliação de follow-up conduzida um ano após o programa. Quando indagados sobre as principais lembranças que tinham do programa, decorridos doze meses após o seu término, todos os adolescentes fizeram referências positivas ao grupo, incluindo: espaço para conversar, receber apoio frente a dificuldades, desabafar, comparar a própria realidade com a de outros adolescentes e se perceber em melhor situação, fazer amizades, aprender ferramentas para subir na vida, aprender a lidar com as pessoas, aprender a lidar com sentimentos, aprender a relaxar, além do prazer em participar. À exceção de um dos entrevistados (que relatou praticar algo, mas não sabia dar nome), todos os demais relataram estar praticando alguma habilidade aprendida no programa. A assertividade, a manifestação de opiniões pessoais, o relaxamento, o controle da raiva e impaciência frente a situações conflituosas, o fazer amizades e a solução de problemas foram as habilidades relatadas pelos participantes cuja prática foi mantida após o término do programa.

Quando questionados sobre o que mudou na vida deles após a participação no grupo que eles julgavam ter relação com o programa, todos os entrevistados mencionaram pelo menos uma mudança positiva, dentre as quais: a redução de conflitos familiares, o aumento do diálogo e da negociação com os pais, a redução da timidez, a construção de novas 
Tabela 2

Avaliação de dose recebida: freqüencia de relatos de prática de ferramentas disponibilizadas na intervenção

\begin{tabular}{|c|c|c|}
\hline Categorias & Freqüência & Porcentagem \\
\hline \multicolumn{3}{|l|}{ Prática de habilidades sociais } \\
\hline Assertividade/dizer não & 24 & 26 \\
\hline Negociar/argumentar com os pais & 15 & 17 \\
\hline Solicitar/dar apoio & 10 & 11 \\
\hline Empatia & 7 & 8 \\
\hline Fazer amizades & 5 & 6 \\
\hline Iniciar conversa & 5 & 6 \\
\hline Controlar raiva e impulsividade & 5 & 6 \\
\hline Reconciliar/pedir desculpa & 5 & 6 \\
\hline Elogiar & 4 & 5 \\
\hline Desabafar/compartilhar & 3 & 3 \\
\hline Expressar opinião & 3 & 3 \\
\hline Expressar emoção/sentimento & 2 & 2 \\
\hline Dar feedback & 2 & 2 \\
\hline Fazer pedido & 1 & 1 \\
\hline Total & 91 & 100 \\
\hline \multicolumn{3}{|l|}{ Prática de estratégias cognitivas } \\
\hline Planejamento solução de problemas & 20 & 32 \\
\hline Planejamento futuro como homem/mulher & 12 & 19 \\
\hline Fortalecimento da auto-eficácia & 11 & 17 \\
\hline Mudança de erros de pensamento & 10 & 16 \\
\hline Auto-observação comportamento & 8 & 13 \\
\hline Leitura do ambiente & 1 & 2 \\
\hline Total & 62 & 100 \\
\hline
\end{tabular}

\section{Prática de enfrentamento ao estresse}

Estratégias de enfrentamento focadas na emoção

\begin{tabular}{lrr} 
Respiração diafragmática & 7 & 58 \\
Relaxamento/concentração & 4 & 33 \\
Ouvir música & 1 & 8 \\
\hline Subtotal & 12 & 100 \\
\hline
\end{tabular}

Estratégias de enfrentamento focadas no problema

\begin{tabular}{lrr} 
Auto-cuidado à saúde & 24 & 69 \\
Iniciativa para solução de problema & 7 & 20 \\
Trabalho remunerado & 2 & 6 \\
Administração do tempo & 1 & 3 \\
Montar grupo de estudo & 1 & 2 \\
\hline Subtotal & 35 & 100 \\
\hline Total & 47 \\
\hline
\end{tabular}

amizades, a redução de estresse e da agressividade, o aumento de habilidades para dizer não e ouvir com empatia, o aumento em habilidades para estabelecer conversação, a maior tolerância a pessoas com pensamentos diferentes, a maior concentração em escolhas profissionais e planos futuros. Sobre dificuldades que se mantiveram após a participação no programa, isto é, não modificadas positivamente pelo programa, três adolescentes relataram: vergonha em dar opiniões, dúvidas quanto às escolhas profissionais, dificuldades em aceitar diferenças e em fazer amizades.

Dos treze entrevistados, sete relataram ter mantido o desempenho escolar, três mencionaram uma melhora no desempenho e três relataram ter piorado o desempenho escolar (redução em notas). Nenhum adolescente relatou ter tido envolvimento com álcool e drogas, ou vivenciado gravidez e doenças sexualmente transmissíveis nos últimos 12 meses 
anteriores à entrevista. Uma das adolescentes mencionou ter experimentado uma redução em sintomas de vitiligo neste período e atribuiu às ferramentas aprendidas no programa um melhor manejo de estresse.

Os pais de três dos adolescentes entrevistados relataram observar a prática das seguintes habilidades em seus filhos: dar opiniões, fazer elogios e controlar a raiva. Os demais, quando questionados sobre habilidades praticadas pelos filhos, relataram não saber identificar a habilidade. Entretanto, todos os pais relataram ter observado mudanças comportamentais nos filhos após a participação no programa, incluindo a redução em conflitos familiares e o aumento em habilidades para manejar raiva, para fazer amizades, para manejar estresse e controlar sintomas psicossomáticos (vitiligo). Um dos pais relatou que a filha permanecia intolerante frente às diferenças e mantinha dificuldade em fazer amizades. Estes dados se mostraram coerentes com aqueles relatados pelos adolescentes acerca das mudanças auto-observadas.

Os relatos dos pais confirmaram o dos filhos no que diz respeito ao desempenho escolar, com manutenção, melhora e piora nas notas, e ao estado de saúde, com ausência de envolvimento com álcool e drogas, de gravidez e de doenças sexualmente transmissíveis.

Tanto os pais quanto os adolescentes foram questionados sobre sugestões para melhoria do programa. Os pais sugeriram o aumento no número de sessões destinadas aos pais, com maior ênfase no fortalecimento dos vínculos familiares. Sugeriram ainda que os pais fossem melhor informados sobre as habilidades trabalhadas na intervenção e que, ao final do programa, houvesse um feedback para os pais acerca dos resultados alcançados pelos filhos. Os adolescentes sugeriram fazer sessões mais longas que 90 minutos, fazer um novo encontro para reunir todos os participantes e incluir o tema da escolha profissional no conteúdo do programa.

\section{Considerações Finais}

A avaliação de processo e resultados do programa aponta evidências favoráveis à efetividade do programa, de acordo com a percepção dos adolescentes e de seus pais, bem como coerência entre os dados da avaliação de processo e de resultados. Durante a intervenção, a maioria das metas intermediárias previstas para o programa teve uma alta ocorrência, os participantes relataram praticar diversas habilidades e demonstraram satisfação frente às sessões. Um ano após o término do programa, nenhum dos adolescentes relatou ter tido envolvimento com álcool e drogas, gravidez e doenças sexualmente transmissíveis, ainda que alguns tivessem percebido uma piora no desempenho escolar, o que foi coerentemente relatado pelos participantes e por seus pais. Além disto, todos os adolescentes relataram ter vivenciado mudanças positivas percebidas como favorecidas pela participação na intervenção, como melhoria nas relações interpessoais na família e grupo de pares, e maior dedicação à construção de projetos de futuro. Os relatos dos pais acerca de mudanças nos filhos foram coerentes com os dos filhos, no que se refere à redução em conflitos familiares e ao desenvolvimento de habilidades de manejo da raiva, do estresse e de construção de novas amizades. A literatura sobre prevenção e promoção de saúde na adolescência (Williams, Holmbeck, \& Greenley, 2002) indica que comportamentos de risco à saúde na adolescência podem ter um efeito duradouro no ciclo de vida levando à morbidade e mortalidade tardias (exemplo: na adolescência podem ter início práticas de abuso de álcool e fumo, o que contribui para o desenvolvimento de diversas doenças crônicas na meia-idade e na velhice). $\mathrm{Na}$ face oposta desta discussão, o desenvolvimento de comportamentos de proteção à saúde na adolescência pode também ter um efeito positivo duradouro nas etapas seguintes da vida. Neste contexto, salienta-se a relevância social dos resultados obtidos no presente estudo.

À exceção da piora no desempenho escolar de três dos participantes, que merece investigação mais aprofundada em novos estudos, os resultados encontrados foram na direção esperada, isto é, de construção de fatores de proteção relativos a competências individuais para enfrentamento às demandas cotidianas. Estes dados estão em acordo com os encontrados por Gorayeb et al. (2003) e Minto et al. (2006), obtidos em programas de habilidades de vida conduzidos com adolescentes em escolas. Pode-se deduzir que o conteúdo, formato e atuação dos facilitadores dos grupos foram adequados no presente estudo. Neste sentido, sugere-se que estudos futuros incluam medidas de integridade ou fidelidade do tratamento, a fim de averiguar se a implementação do conteúdo da intervenção foi fiel ao planejamento feito. Um outro aspecto a se considerar foi a predominância de relatos de práticas de habilidades sociais e mudanças nas relações interpessoais familiares e entre pares, em comparação com menos relatos de mudanças em estratégias cognitivas e de enfrentamento ao estresse. Isto pode ter resultado do conteúdo da intervenção, que incluiu um número maior de sessões sobre habilidades sociais em comparação à quantidade de sessões acerca das habilidades cognitivas e de manejo de estresse. Intervenções futuras podem reorganizar este conteúdo, com ampliação das discussões sobre estas duas últimas classes de habilidades de vida.

Ainda que positivos em seu conjunto, os dados encontrados devem ser vistos com cautela, por algumas razões. A primeira é que nem todos os adolescentes participantes da intervenção foram entrevistados durante a avaliação um ano depois. Portanto, não se sabe qual o impacto do programa para os cinco adolescentes que não foram localizados nesta etapa da avaliação. Isto nos impede de afirmar que o programa foi benéfico para todos os participantes e que nenhum deles apresentou os problemas que o programa buscava prevenir. Uma segunda razão é que a efetividade de programas preventivos só pode ser constatada em sucessivas avaliações longitudinais. Logo, novas avaliações deste grupo de intervenção, ao longo dos anos da adolescência, são necessárias para se verificar se o programa previne de fato aquilo que se propõe a definir. Uma terceira razão é que não se sabe em que extensão as mudanças constatadas um ano depois são, de fato, devidas ao programa, uma vez que não se contou com um grupo comparação ou grupo controle. Por outro lado, os dados de avaliação de processo e follow-up minimizam esta limitação ao apontarem coerência entre o andamento da intervenção e seus resultados finais. Ainda assim, delineamentos capazes de gerar evidências mais sólidas de efetividade se 
fazem necessários em novos estudos, seja por meio da quaseexperimentação ou experimentação (Pentz, 2004) ou por meio de análises de mudança confiável e significância clínica (Del Prette \& Del Prette, 2008). Uma quarta consideração é que os dados encontrados resultam de uma intervenção em uma amostra pequena e muito específica, exposta a diversos fatores de proteção, como ter pais instruídos e participar de atividades grupais e esportivas. É preciso replicar o programa junto a diversas populações, incluindo aquelas mais expostas a riscos pessoais e sociais, e verificar se os resultados aqui encontrados se repetem ou não.

Um dos principais aspectos a ser aprimorado em versões futuras deste trabalho é o módulo destinado à família. Como sugerido pelos pais durante a entrevista final, seria desejável um incremento na intervenção para os pais, incluindo o aumento em número de horas de intervenção, a discussão detalhada das várias habilidades ensinadas no programa e os procedimentos dos pais para favorecer a generalização destas habilidades. Quanto à sugestão de um dos pais para que fosse dado um feedback sobre o crescimento dos filhos, este procedimento foi planejado, mas não ocorreu na prática. Os pais foram informados, através de uma carta, sobre a disponibilidade da equipe em fazer uma entrevista de devolução para os pais e adolescentes, de modo individualizado para cada família. Para tanto, os pais deveriam telefonar e marcar o horário que melhor lhes conviesse. Todavia, nenhum pai procurou a equipe para esta finalidade. É possível que o custo da resposta tenha sido alto para os pais (exemplo: ligar, marcar horário e se deslocar até o local especificado, em meio a uma rotina de vida cheia de compromissos) e que isto tenha impedido a adesão a esta entrevista devolutiva. Uma outra explicação possível é que, por se tratar de um programa preventivo e não de tratamento, os pais tenham visto pouca urgência ou utilidade em tal devolução, já que seus filhos não estavam "doentes".

Considera-se que o presente estudo ofereceu algumas contribuições para o andamento das pesquisas em prevenção em saúde na adolescência. Uma delas se refere ao planejamento da avaliação com inclusão de indicadores de processo e followup, bem como a junção de medidas observacionais e verbais, estas últimas obtidas junto aos adolescentes e seus pais. As medidas de processo usadas foram não invasivas e naturalmente inseridas no contexto da intervenção, o que pode ser considerado vantajoso em estudos de avaliação de intervenção. Um ganho metodológico necessário para o avanço da área seria o acréscimo de medidas quantitativas para avaliações de pré e pós-teste, como escalas de habilidades de vida, que precisam ainda ser desenvolvidas e validadas para o Brasil, e o uso de medidas diretas de notas escolares, como o boletim escolar. Esta sugestão poderia ampliar a lógica da triangulação (Minayo, Assis, \& Souza, 2005), certamente opção promissora em avaliação de programas preventivos em saúde do adolescente. Uma outra contribuição deste estudo é viabilização da replicação, uma vez que o procedimento está descrito, em seus objetivos e técnicas (Murta, 2008). Neste caso, considera-se que seria útil, tanto para fins de pesquisa quanto de serviço, a replicação deste programa em contextos de atenção à saúde e desenvolvimento do adolescente, como escolas, unidades básicas de saúde, unidades do programa de erradicação do trabalho infantil, unidades dos centros de atenção psicossocial à infância e adolescência, programas de atenção integral à família, programas de medidas sócio-educativas, dentre outros.

\section{Referências}

Bolsoni-Silva, A., \& Marturano, E. (2002). Práticas educativas e problemas de comportamento: uma análise a luz das habilidades sociais. Estudos de Psicologia (Natal), 7, 227-235.

Botvin, G. J. (1996). Life skills training: teacher's manual for middle/junior high school. New Jersey: Teacher's Health Press.

Campbell, D. T., \& Stanley, J. C. (1979). Delineamentos experimentais e quase-experimentais de pesquisa (R. A. T. Di Dio, Trad.). São Paulo: EPU e EDUSP.

Coelho, M. V., \& Murta, S. G. (2007). Treinamento de pais em grupo: um relato de experiência. Estudos de Psicologia (Campinas), 24, 333-341.

Del Prette, A., \& Del Prette, Z. A. P. (2001). Psicologia das relações interpessoais: vivencias para o trabalho em grupo. Petrópolis: Vozes.

Del Prette, Z. A. P., \& Del Prette, A. (2008). Significância clínica e mudança confiável na avaliação de intervenções psicológicas. Psicologia: Teoria e Pesquisa, 24, 497-505.

Durlak, J. A., \& Wells, A. M. (1997). Primary prevention mental health programs for children and adolescents: a meta-analytic review. American Journal of Community Psychology, 25, 115-142.

Essau, C. A. (2002). Primary prevention of depression. In D. J. A. Dozois \& K. S. Dobson (Orgs.). The prevention of anxiety and depression: theory, research and practice. (pp. 185-204). Washington: American Psychological Association.

Fernández-Ballesteros, R., Vedung, E., \& Seyfried, E. (1998). Psychology in program evaluation. European Psychologist, 3, 143-154.

Gomide, P. I. C. (2003). Estilos parentais e comportamento anti-social. In A. Del Prette e Z. Del Prette (Orgs.). Habilidades sociais, desenvolvimento e aprendizagem: questões conceituais, avaliação e intervenção (pp. 21-60). Campinas: Alínea.

Gorayeb, R. (2002). O ensino de habilidades de vida em escolas no Brasil. Psicologia, Saúde \& Doença, 3, 213-217.

Gorayeb, R., Cunha Netto, J. R., \& Bugliani, M. A. P. (2003). Promoção de saúde na adolescência: experiência com programas de ensino de habilidades de vida. In Z. A. Trindade \& A. N. Andrade (Orgs.). Psicologia e saúde: um campo em construção (pp.89-100). São Paulo: Casa do Psicólogo.

Hawkins, J. D., Catalano, R. F. \& Miller, J. Y. (1992). Risk and protective factors for alcohol and other drug problems in adolescence and early adulthood: implications for substance abuse prevention. Psychological Bulletin, 112, 64-105.

Kholenberg, R. J., \& Tsai, M. (2001). Psicoterapia analítica functional: criando relações terapêuticas intensas e curativas ( $\mathrm{R}$. Kerbauy, Trad.). Santo André: ESETec.

Kulic, K. R., Horne, A. M., \& Dagley, J. C. (2004). A comprehensive review of prevention groups for children and adolescents. Group Dynamics: Theory, Research and Practice, 8, 139-151.

Liebman, M. (2000). Exercícios de arte para grupos: um manual de temas, jogos e exercícios. (R. Migliorini, Trad.). São Paulo: Summus.

Mangrulkar, L., Whitman, C. V., \& Posner, M. (2001). Enfoque de habilidades para la vida para un desarrollo saludable de niños y adolescentes. Washington: Organización Panamericana de la Salud.

McCarter, A. K., Sowers, K. M., \& Dulmus, C. N. (2004). Adolescent suicide prevention. In L. A. Rapp-Paglicci, C. N. Dulmus \& J. S. Wodarski (Orgs.). Handbook of preventive interventions for children and adolescents (pp. 8599). New Jersey: John Willey \& Sons. 
Meschke, L. L., \& Patterson, J. M. (2003). Resilience as a theoretical basis for substance abuse prevention. The Journal of Primary Prevention, 23, 483-514.

Minayo, M. C. S., Assis, S. G., \& Souza, E. R. (2005). Avaliação por triangulação de métodos: abordagem de programas sociais. Rio de Janeiro: FIOCRUZ.

Minto, E. C., Pedro, C. P., Netto, J. R. C, Bugliani, M. A. P., \& Gorayeb, R. (2006). Ensino de habilidades de vida na escola: uma experiência com adolescentes. Psicologia em Estudo, 11(3), 561-568.

Murta, S. G. (2007a). Programas de prevenção a problemas emocionais e comportamentais em crianças e adolescentes: lições de três décadas de pesquisa. Psicologia: Reflexão e Crítica, 20, 4-11.

Murta, S. G. (2007b). Avaliação de processo de um programa de manejo de estresse ocupacional. Psicologia: Reflexão e Crítica, 20, 295-302.

Murta, S. G. (2008). Programa de habilidades de vida para adolescentes: um manual para aplicadores. Goiânia: Porã Cultural.

Murta, S. G., Del Prette, A., Nunes, F. C., \& Del Prette, Z. A. P. (2007). Problemas en la adolescencia: contribuciones del entrenamiento en habilidades sociales. In M. R. R. Saldana (Org.), Intervención psicológica para adolescentes: Ámbitos educativo y de la salud. Bogotá: PSICOM Editores.

Murta, S. G., Sanderson, K., \& Oldenburg, B. (2007). Process evaluation in occupational stress management programs: A systematic review. American Journal of Health Promotion, 21, 248-254.

Pentz, M. A. (2004). Form follows function: designs for prevention effectiveness and diffusion research. Prevention Science, 5, 23-29.

Pinheiro, M. I. S., Haase, V. G., Del Prette, A., Amarente, C. L. D., \& Del Prette, Z. A. P. (2006). Treinamento de habilidades sociais educativas para pais de crianças com problemas de comportamento. Psicologia: Reflexão e Crítica, 19, 407-414.

Posavac, E. J., \& Carey, R. G. (2003). Program evaluation. Methods and case studies. New Jersey: Prentice Hall.

Shochet, I. M.; Dadds, M. R.; Holland, D.; Whitefield, K.; Harnett, P. H., \& Osgarby, S. M. (2001). The efficacy of a universal school-based program to prevent adolescent depression. Journal of Clinical Child Psychology, 30, 303-315.

Serrão, M., \& Baleeiro, M. C. (1999). Aprendendo a ser e a conviver. São Paulo: FTD.

Spence, S. H. (2003). Social skill training with children and young people: theory, evidence and practice. Child and Adolescent Mental Health, 8, 84-96.

Stallard, P. (2004). Bons pensamentos, bons sentimentos: manual de terapia cognitivo-comportamental para crianças e adolescentes (C. A. S. N. Soares, Trad.). Porto Alegre: Artmed.

Steckler, A., \& Linnan, L. (2002). Process evaluation for public health interventions and research: an overview. In A. Steckler \& L. Linnan (Orgs.), Process evaluation for public health interventions and research (pp. 1-21). San Francisco, CA: Jossey-Bass.

Straub, R. (2005). Psicologia da Saúde (R. C. Costa, Trad.). Porto Alegre: ArtMed.

Virgolim, A. M. R., Fleith, D. S., \& Neves-Pereira, M. S. (1999). Toc, troc... plim, plim! Lidando com as emoções, brincando com o pensamento através da criatividade. Campinas: Papirus.

Williams, P. G., Holmbeck, G. N., \& Greenley, R. N. (2002). Adolescent health psychology. Journal of Consulting and Clinical Psychology, 70, 828-842.

Sheila Giardini Murta, doutora em Psicologia Social e do Trabalho pela Universidade de Brasília (UnB), é Professora Adjunta do Departamento de Psicologia Clínica - Instituto de Psicologia (UnB). Endereço para correspondência: Universidade de Brasília, Campus Universitário Darcy Ribeiro, ICC Norte, IP, PCL. Brasília, DF. CEP: 70910-900.

Fones: (61) 9633 9631, (61) 33072625 ramal 315, FAX (61) 33477746.

E-mail: giardini@unb.br; murta@cultura.com.br

Francimara Azevedo Borges, especialista em Análise e Diagnóstico Organizacional pela Pontifícia Universidade Católica de Goiás (PUCGO), é Analista de Recursos Humanos.

E-mail: francimaraborges@yahoo.com.br

Danilo Cruvinel Ribeiro, é psicólogo pela Pontifícia Universidade Católica de Goiás (PUCGO). E-mail:cruvinelpsi@gmail.com

Eliana Porto Rocha, psicóloga pela Pontifícia Universidade Católica de Goiás (PUCGO), é especializanda em Juventude no Mundo Contemporâneo pela Faculdade Jesuíta de Filosofia (FAJE/ Belo Horizonte /MG). E-mail: elianaporto@hotmail.com

Jordana Calil Lopes de Menezes, psicóloga pela Pontifícia Universidade Católica de Goiás (PUCGO), é especializanda em Getalterapia pela Pontifícia Universidade Católica de Goiás (PUCGO). E-mail: jordanacalil@yahoo.com.br

Marina de Moraes e Prado, especialista em Psicologia Clínica pela Pontifícia Universidade Católica do Rio Grande do Sul (PUCRS), é mestranda em Psicologia Clínica pela Pontifícia Universidade Católica do Rio Grande do Sul (PUCRS). E-mail: marinademoraes@gmail.com 